\title{
A Hybrid Estimation Framework for Cooperative Localization under Communication Constraints
}

\author{
Esha D. Nerurkar, Ke X. Zhou, and Stergios I. Roumeliotis
}

\begin{abstract}
In this paper, we consider the problem of multicentralized Cooperative Localization (CL) under severe communication constraints, i.e., when each robot can communicate only a single bit per real-valued (analog) measurement. Existing approaches, such as those based on the Sign-of-Innovation Kalman filter (SOI-KF) and its variants, require each robot to process quantized versions of both its local (i.e., recorded by its own sensors) and remote (i.e., collected by other robots) measurements. This results in suboptimal performance since each robot has to discard information that is available in its own analog measurements. To address this limitation, we introduce a novel hybrid estimation scheme that enables each robot to process both quantized (from remote sensors) and analog (from its own sensors) measurements. Specifically, we first present the hybrid (H)-SOI-KF, a direct extension of the SOI-KF, for processing both types of measurements. Secondly, we introduce the modified (M)H-SOI-KF, that uses an asymmetric encoding/decoding scheme to incorporate additional information during quantization (based on the hybrid estimates locally available to each robot), resulting in substantial accuracy improvement. Lastly, we present extensive simulations which demonstrate that both hybrid estimators not only outperform the SOI-KF, but also achieve accuracy comparable to that of the standard (analog) centralized Kalman filter.
\end{abstract}

\section{INTRODUCTION AND RELATED WORK}

For robot teams navigating in GPS-denied environments (e.g., space and underwater exploration [1], search and rescue missions [2], and surveillance [3]), an alternative approach for accurate multi-robot pose (i.e., position and orientation) determination is Cooperative Localization (CL) [4]. In CL, instead of localizing independently, groups of communicating robots use their relative measurements (e.g., distance, bearing, and orientation) to jointly estimate their poses, resulting in increased accuracy for the entire team.

Communication-bandwidth considerations play a pivotal role in CL since each robot has to share its own information, either in the form of its pose estimate and covariance, or its exteroceptive and proprioceptive measurements, with other robots in the team [5], [6]. However, factors such as: (1) inherent communication-bandwidth limitations in space and underwater applications, or (2) constraints imposed on data transmission to conserve battery power and increase the robots' longevity in search and rescue operations, prevent robots from transmitting all the required information. Various approaches that address this issue of resource-constrained CL

This work was supported by the University of Minnesota (DTC), and the National Science Foundation (EIA-0324864, IIS-0643680, IIS-0811946)

E. D. Nerurkar, and S. I. Roumeliotis are with the Department of Computer Science and Engineering, Univ. of Minnesota, Minneapolis, USA \{nerurkar, stergios\}@cs.umn. edu

K. X. Zhou is with the Department of Electrical and Computer Engineering, Univ. of Minnesota, Minneapolis, USA kezhoudece . umn . edu have been proposed in the literature. For instance, in some cases robots select and transmit a subset of their real-valued ${ }^{1}$ (analog) measurements depending on the communication bandwidth availability [7], [8]. However, in this paper we focus on CL under severe communication constraints where each robot can transmit only a single bit per real-valued measurement. As a result, every robot has to quantize each of its analog measurements before communicating them to the team.

Estimation using quantized measurements, in the context of wireless sensor networks, has received considerable attention in the signal processing community [9], [10], [11]. Recently, a quantized-innovation filter for estimating stochastic dynamical processes has been proposed [12], where the measurement innovation, instead of the actual real-valued measurement, is quantized to a single bit. The proposed Sign-of-Innovation Kalman filter (SOI-KF) is derived for linear Gaussian process and measurement models and it approximates the posterior probability density function (pdf) by a Gaussian ${ }^{2}$, after each measurement update. This results in a linear state-update equation for the SOI-KF, whose structure is strikingly similar to that of the standard Kalman filter. When multiple bits are available for quantizing a measurement, the SOI-KF approach has been extended to the batch-quantized KF (BQ-KF) and the iteratively-quantized KF (IQ-KF) [13]. An extension of this approach to the batch Maximum A Posteriori (MAP) estimation framework, the quantized MAP (Q-MAP) estimator, is described in [14].

An advantage of the multi-centralized architecture, used by all the quantized-innovation estimation schemes discussed above, is its robustness to single-point failures. In multicentralized CL (MC-CL), each robot broadcasts all its measurements to the team and every robot locally processes these measurements to generate pose estimates for the entire team. However, since the quantization rules for these schemes depend on the state estimates, it forces all robots to maintain identical estimators to ensure consistency. Hence, each robot is restricted to use quantized versions of its own locallyavailable, analog measurements for estimation. Thus, valuable information, that can be used to reduce the uncertainty of the pose estimates, is discarded.

In this paper, our objective is to devise an estimation framework that enables each robot to incorporate its locally-

\footnotetext{
${ }^{1}$ Sensors sample a process and provide a measurement which is often represented in digital form using 32 or 64 bit floating-point number representation. We refer to such measurements as analog or real-valued.

${ }^{2}$ Note that due to the non-linearity of the quantization operation, the posterior pdf is not, in general, a Gaussian.
} 
available analog measurements, in order to improve the accuracy of CL. To achieve this, we propose a hybrid estimation framework wherein each robot maintains two local estimators (see Fig. 1): (i) a quantized (Q) estimator that processes quantized measurements from all robots, including itself, and (ii) a hybrid $(\mathrm{H})$ estimator that processes its own analog measurements along with the quantized measurements from other robots in the team. Firstly, we present the H-SOI-KF, i.e., the $\mathrm{H}$-estimator based on the SOI quantization rule [12]. Note that the existing SOI-KF is the corresponding Q-estimator for this quantization rule. We validate the performance of the H-SOI-KF in simulation and show that it outperforms the SOI-KF in terms of estimation accuracy.

Secondly, we demonstrate that the accuracy of the hybrid estimation scheme can be further improved by incorporating information from the H-estimator into the quantization rule. In contrast to the SOI quantization rule, where the quantization threshold depends only upon the estimates generated by the Q-estimator (SOI-KF), we postulate a new (modified) quantization rule that uses information from both the $\mathrm{H}$ estimator and the Q-estimator. Next, we prove that the quantized bit generated using this new rule is more informative than the SOI bit. Furthermore, subject to communication constraints, we derive the modified Q-estimator ((M)-SOI$\mathrm{KF})$ and modified $\mathrm{H}$-estimator $((\mathrm{M}) \mathrm{H}-\mathrm{SOI}-\mathrm{KF})$ for this new quantization rule. Lastly, we present extensive simulation studies to show that the estimates generated by these modified estimators are significantly more accurate than even the $\mathrm{H}-\mathrm{SOI}-\mathrm{KF}$ estimates, and furthermore, their performance is comparable to that of a centralized Kalman filter processing all analog measurements.

\section{Problem Formulation}

Consider a team of $N$ robots navigating in $2 \mathrm{D}$ while performing MC-CL. We assume that the system and measurement models are known a priori to all robots and the robots' communication graph is complete (i.e., each robot can communicate with all robots in the team at every time step). The process and measurement models for the robot team are given by the following linear discrete-time dynamic system (for $i=1, \ldots, N$, and $m=1, \ldots, M_{k}^{i}$ )

$$
\begin{aligned}
\mathbf{x}_{k} & =\mathbf{F}_{k-1} \mathbf{x}_{k-1}+\mathbf{G}_{k-1} \mathbf{w}_{k-1}, \mathbf{x}_{0} \sim \mathcal{N}\left(\mathbf{x}_{i n i t}, \mathbf{P}_{\mathbf{0}}\right) \\
z_{k m}^{i} & =\mathbf{h}_{k m}^{i^{T}} \mathbf{x}_{k}+v_{k m}^{i}
\end{aligned}
$$

with zero-mean, white Gaussian and uncorrelated system and measurement noise with covariance

$$
\begin{aligned}
& E\left[\mathbf{w}_{k} \mathbf{w}_{l}^{T}\right]=\delta_{k l} \mathbf{Q}_{k}, E\left[v_{k m}^{i} v_{l n}^{i}\right]=\delta_{k l, m n} \sigma_{k m}^{i^{2}} \\
& E\left[v_{k m}^{i} v_{l n}^{j}\right]=0, \forall j \neq i, j=1, \ldots, N .
\end{aligned}
$$

Here, $\quad \mathbf{x}_{k}=\left[\mathbf{x}_{k}^{1^{T}}, \mathbf{x}_{k}^{2^{T}}, \ldots, \mathbf{x}_{k}^{N^{T}}\right]^{T} \quad$ where, $\mathbf{x}_{k}^{i}=\left[x_{k}^{i}, y_{k}^{i}, \phi_{k}^{i}\right]^{T}$, is the state of robot $i$, which generates $M_{k}^{i}$ scalar measurements at time-step $k$.

In this formulation, we assume a statistical motion model (e.g., the constant-velocity model [15]), in which the only control input is the system noise (see (1)). This allows us to treat both proprioceptive (linear and rotational velocity) and exteroceptive (relative distance/bearing/orientation) measurements, identically. Furthermore, a single vector-valued measurement can be decomposed into multiple scalar measurements using pre-whitening and then processed using the above formulation. This linear formulation is used only to aid in the mathematical derivation. For real-world examples, the linearized system, obtained from the underlying non-linear system and measurement models, will be used.

\section{A. Real vs. Quantized Measurements}

In the absence of communication-bandwidth constraints, the Minimum Mean Squared Error (MMSE) estimate of the robots' poses at time-step $k, \hat{\mathbf{x}}_{k \mid k}$, given all real-valued measurements up to time-step $k, \mathbf{z}_{0: k}$, is calculated as

$$
\hat{\mathbf{x}}_{k \mid k}=E\left[\mathbf{x}_{k} \mid \mathbf{z}_{0: k}\right]=\int_{\mathbb{R}^{p}} \mathbf{x}_{k} p\left[\mathbf{x}_{k} \mid \mathbf{z}_{0: k}\right] d \mathbf{x}_{k}, \quad \mathbf{x}_{k} \in \mathbb{R}^{p} .
$$

Here, $p=3 N$ and $\mathbf{z}_{0: k}=\left[\left(\mathbf{z}_{0: k}^{1}\right)^{T}, \ldots,\left(\mathbf{z}_{0: k}^{N}\right)^{T}\right]^{T}$ where, $\mathbf{z}_{0: k}^{i}=\left[\left(\mathbf{z}_{0}^{i}\right)^{T}, \ldots,\left(\mathbf{z}_{k}^{i}\right)^{T}\right]^{T}$ and $\mathbf{z}_{l}^{i}=\left[z_{l 1}^{i}, \ldots, z_{l M_{l}^{i}}^{i}\right]^{T}$, $l=0, \ldots, k$. Under the Gaussian noise assumption for the linear system (1)-(3), the prior pdf, $p\left[\mathbf{x}_{k} \mid \mathbf{z}_{0: k-1}\right]$, and the posterior pdf, $p\left[\mathbf{x}_{k} \mid \mathbf{z}_{0: k}\right]$, are completely characterized by their mean and covariance and can be obtained using the standard Kalman filter (see Algorithm 1). Note that the realvalued measurements, $z_{k m}^{i}$, are required for updating the state in the Kalman filter and have to be broadcasted by robot $i$ to the entire team.

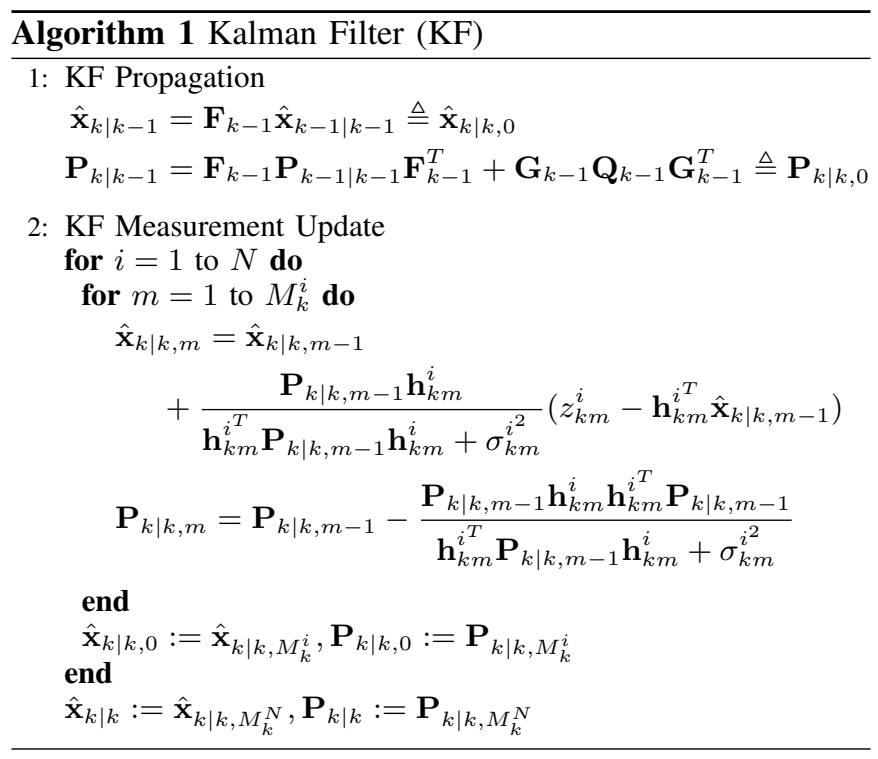

On the contrary, consider the scenario where each robot can broadcast only a single bit, $b_{k m}^{i}$, per analog measurement, $z_{k m}^{i}$. The MMSE estimate of the robots' poses, $\hat{\mathbf{x}}_{k \mid k}^{Q}$, given these quantized measurements, $\mathbf{b}_{0: k}$, from all robots up to time-step $k$ is calculated as

$$
\hat{\mathbf{x}}_{k \mid k}^{Q}=E\left[\mathbf{x}_{k} \mid \mathbf{b}_{0: k}\right]=\int_{\mathbb{R}^{p}} \mathbf{x}_{k} p\left[\mathbf{x}_{k} \mid \mathbf{b}_{0: k}\right] d \mathbf{x}_{k}, \quad \mathbf{x}_{k} \in \mathbb{R}^{p} .
$$




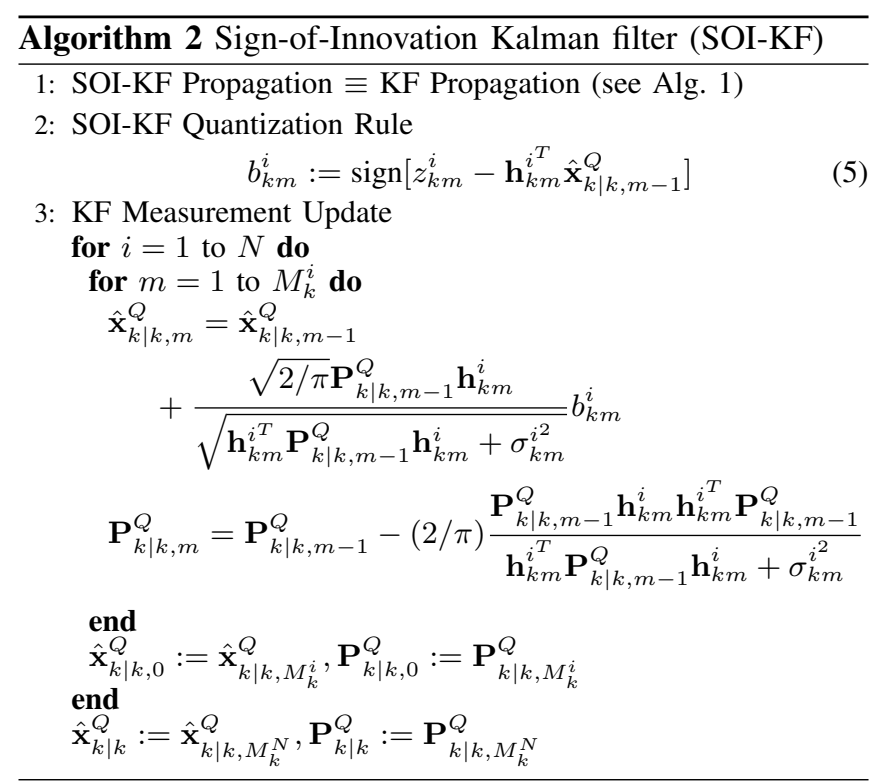

Note that due to quantization, the prior and posterior pdfs are no longer Gaussian even for linear process and measurement models. Moreover, the computation of these non-Gaussian pdfs is, in general, intractable. The SOI-KF [12], where $b_{k m}^{i}$ is chosen to be the quantized measurement innovation (see quantization rule in Algorithm 2), simplifies this computation by approximating these pdfs as Gaussians (similar to the Extended Kalman filter ${ }^{3}$ ). Note that the resulting estimator has a structure very similar to that of the Kalman filter (see Algorithm 1). In Fig. 1, when switch $\mathrm{S} 1$ is in position 1 but the shaded Hybrid Estimators do not exist, we obtain the SOI-KF.

However, a shortcoming of the SOI-KF is that even though each robot $i$ has access to its own analog measurements, $\mathbf{z}_{0: k}^{i}$, the SOI-KF forces it to discard this information and process only the corresponding quantized innovations, $\mathbf{b}_{0: k}^{i}$. This is necessary because the quantization threshold, which is set to be the predicted measurement, $\mathbf{h}_{k m}^{i^{T}} \hat{\mathbf{x}}_{k \mid k, m-1}^{Q}$, has to be identical for every robot to ensure consistent estimation. This in turn implies that all robots have to process the same set of measurements, so that they can generate identical state estimates, $\hat{\mathbf{x}}_{k \mid k, m-1}^{Q}$.

\section{HYBRID ESTIMATION FRAMEWORK}

We now present a hybrid estimation framework that enables each robot to incorporate its analog measurements in the estimation process to improve the accuracy of CL. In our proposed approach, each robot $i$ maintains two estimators (see Fig. 1): (1) a quantized (Q) estimator that processes quantized measurements from all robots including itself, i.e., $\hat{\mathbf{x}}_{k \mid k}^{Q}=E\left[\mathbf{x}_{k} \mid \mathbf{b}_{0: k}^{q \neq i}, \mathbf{b}_{0: k}^{i}\right]$, and (2) a hybrid (H) estimator that processes its own real-valued measurements and quantized measurements from the other robots in the

\footnotetext{
${ }^{3}$ It is important to note that this loss of Gaussianity in the EKF is due to the non-linearity of the process and measurement models as opposed to the non-linearity of the quantization step that we discuss here.
}

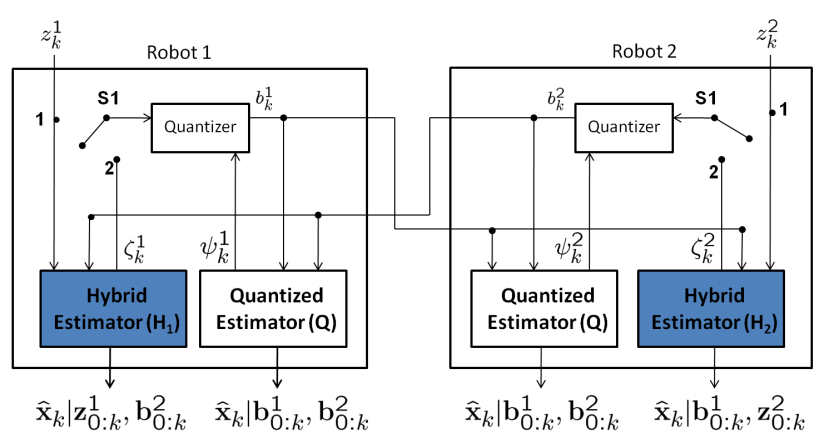

Fig. 1. Hybrid Estimation framework. Here, $\psi_{k}^{i}=h_{k}^{i^{T}} \hat{\mathbf{x}}_{k \mid k-1}^{Q}$ and $\zeta_{k}^{i}=$ $h_{k}^{i^{T}} \hat{\mathbf{x}}_{k \mid k}^{H_{i}}, i=1,2$. When switch $\mathrm{S} 1$ is in position 1 , we obtain the hybrid estimation scheme corresponding to the SOI quantization rule where the Quantized estimator corresponds to the SOI-KF and the Hybrid estimator corresponds to the H-SOI-KF. Now, when switch S1 is in position 2, we obtain the hybrid estimation scheme corresponding to the new quantization rule in (16) where the Quantized estimator corresponds to the M-SOI-KF and the Hybrid estimator corresponds to the MH-SOI-KF.

team, i.e., $\hat{\mathbf{x}}_{k \mid k}^{H_{i}}=E\left[\mathbf{x}_{k} \mid \mathbf{b}_{0: k}^{q \neq i}, \mathbf{z}_{0: k}^{i}\right], q=1, \ldots, N$. We highlight that the estimates generated by the Q-estimator are identical for all robots since each robot processes identical measurements, $\mathbf{b}_{0: k}=\left\{\mathbf{b}_{0: k}^{q \neq i}, \mathbf{b}_{0: k}^{i}\right\}$. However, the estimates generated by the H-estimator are different for each robot, since each robot processes a different set of quantized and analog measurements.

In this section, we first derive the H-estimator, H-SOI$\mathrm{KF}$, based on the SOI quantization rule and establish that it generates more accurate estimates than the SOI-KF. Next we demonstrate that the performance of the hybrid estimation framework can be further improved by incorporating the estimates from the $\mathrm{H}$-estimator into the quantization rule.

\section{A. SOI Quantization Rule (H-SOI-KF)}

For the hybrid estimation framework based on the SOI quantization rule, the Q-estimator is identical to the SOIKF by definition. The H-estimator, H-SOI-KF, is obtained as follows.

Proposition 1: H-SOI-KF

Consider the linear model of (1)-(3). If robot $i$ assumes that the pdf $p\left[\mathbf{x}_{k} \mid \mathbf{b}_{0: k, m-1}^{q \neq i}, \mathbf{z}_{0: k, m-1}^{i}\right]$ is Gaussian with $\mathcal{N}\left(\hat{\mathbf{x}}_{k \mid k, m-1}^{H_{i}}, \mathbf{P}_{k \mid k, m-1}^{H_{i}}\right)$, then the MMSE estimator for processing the analog measurement, $z_{k m}^{i}$, is identical to the Kalman filter (see Algorithm 1). On the other hand, for the MMSE estimator that processes the quantized measurement from robot $j, b_{k m}^{j}, j \neq i$, the state and covariance propagation equations are identical to the Kalman filter, while the state and covariance update equations are given by

$$
\begin{gathered}
\hat{\mathbf{x}}_{k \mid k, m}^{H_{i}}=\hat{\mathbf{x}}_{k \mid k, m-1}^{H_{i}}+\frac{(\alpha / \sqrt{2 \pi}) \mathbf{P}_{k \mid k, m-1}^{H_{i}} \mathbf{h}_{k m}^{j}}{\sqrt{\mathbf{h}_{k m}^{j^{T}} \mathbf{P}_{k \mid k, m-1}^{H_{i}} \mathbf{h}_{k m}^{j}+\sigma_{k m}^{j^{2}}}} b_{k m}^{j} \\
\mathbf{P}_{k \mid k, m}^{H_{i}}=\mathbf{P}_{k \mid k, m-1}^{H_{i}}-\frac{\alpha^{2} \mathbf{P}_{k \mid k, m-1}^{H_{i}} \mathbf{h}_{k m}^{j} \mathbf{h}_{k m}^{j^{T}} \mathbf{P}_{k \mid k, m-1}^{H_{i}}}{2 \pi\left(\mathbf{h}_{k m}^{j^{T}} \mathbf{P}_{k \mid k, m-1}^{H_{i}} \mathbf{h}_{k m}^{j}+\sigma_{k m}^{j^{2}}\right)}
\end{gathered}
$$


where

$$
0 \leq \alpha=\frac{\exp \left[-\beta^{2} / 2\right]}{\mathrm{Q}\left[b_{k m}^{j} \beta\right]}
$$

and

$$
\beta=\frac{\mathbf{h}_{k m}^{j^{T}}\left(\hat{\mathbf{x}}_{k \mid k, m-1}^{Q}-\hat{\mathbf{x}}_{k \mid k, m-1}^{H_{i}}\right)}{\sqrt{\mathbf{h}_{k m}^{j^{T}} \mathbf{P}_{k \mid k, m-1}^{H_{i}} \mathbf{h}_{k m}^{j}+\sigma_{k m}^{j^{2}}}}
$$

Proof: See Appendix.

Thus, even though each robot cannot communicate its analog measurements to the team, the H-SOI-KF enables it to process these measurements locally and obtain state estimates that are more accurate than the SOI-KF (see Fig. 2).

Note than in the SOI quantization rule, the H-SOI-KF estimates generated by each robot do not play any role in computing the quantization threshold, which depends solely on $\hat{\mathbf{x}}_{k \mid k, m-1}^{Q}$. However, since the H-SOI-KF estimates are more accurate, by including these estimates in the quantization rule, we can improve its accuracy. Based on this observation, we propose a general linear quantization rule that uses information from both the Q-estimator and $\mathrm{H}$ estimator for determining the quantization threshold. We hypothesize that this new rule will improve the estimation accuracy of both these estimators.

\section{B. General Quantization Rule (MH-SOI-KF)}

We begin by assuming a general linear quantization rule where robot $j$ generates bit $b_{k m}^{j}$, corresponding to the real measurement $z_{k m}^{j}$, as follows

$$
b_{k m}^{j}:=\operatorname{sign}\left[z_{k m}^{j}+\mathbf{d}^{T} \hat{\mathbf{x}}_{k \mid k, m-1}^{H_{j}}+\mathbf{c}^{T} \hat{\mathbf{x}}_{k \mid k, m-1}^{Q}\right]
$$

where $\mathbf{d}$ and $\mathbf{c}$ are deterministic design parameters. Note that by setting $\mathbf{d}=\mathbf{0}$ and $\mathbf{c}=-\mathbf{h}_{k m}^{j}$ in (9), we obtain the SOI quantization rule. We now present the state and covariance update equations for the $\mathrm{Q}$ and $\mathrm{H}$-estimators designed using (9), noting that the state and covariance propagation equations remain identical to those of the Kalman filter.

Proposition 2: Q-estimator

Consider the linear model of (1)-(3). If $p\left[\mathbf{x}_{k} \mid \mathbf{b}_{0: k, m-1}\right]$ is assumed to be Gaussian with $\mathcal{N}\left(\hat{\mathbf{x}}_{k \mid k, m-1}^{Q}, \mathbf{P}_{k \mid k, m-1}^{Q}\right)$, then the state and covariance update equations for the MMSE estimator that processes bit $b_{k m}^{j}$, for all robots is given by

$$
\begin{gathered}
\hat{\mathbf{x}}_{k \mid k, m}^{Q}=\hat{\mathbf{x}}_{k \mid k, m-1}^{Q}+\frac{\left(\alpha_{Q} / \sqrt{2 \pi}\right) \mathbf{P}_{k \mid k, m-1}^{Q} \mathbf{h}_{k m}^{j}}{\sqrt{\mathbf{h}_{k m}^{j^{T}} \mathbf{P}_{k \mid k, m-1}^{Q} \mathbf{h}_{k m}^{j}+\sigma_{k m}^{j^{2}}}} b_{k m}^{j} \\
\mathbf{P}_{k \mid k, m}^{Q}=\mathbf{P}_{k \mid k, m-1}^{Q}-\frac{\alpha_{Q}^{2} \mathbf{P}_{k \mid k, m-1}^{Q} \mathbf{h}_{k m}^{j} \mathbf{h}_{k m}^{j^{T}} \mathbf{P}_{k \mid k, m-1}^{Q}}{2 \pi\left(\mathbf{h}_{k m}^{j^{T}} \mathbf{P}_{k \mid k, m-1}^{Q} \mathbf{h}_{k m}^{j}+\sigma_{k m}^{j^{2}}\right)}
\end{gathered}
$$

where

$$
0 \leq \alpha_{Q}=\frac{\exp \left[-\beta_{Q}^{2} / 2\right]}{\mathrm{Q}\left[b_{k m}^{j} \beta_{Q}\right]}
$$

and

$$
\beta_{Q}=\frac{-\mathbf{d}^{T} \hat{\mathbf{x}}_{k \mid k, m-1}^{H_{j}}-\left(\mathbf{c}^{T}+\mathbf{h}_{k m}^{j^{T}}\right) \hat{\mathbf{x}}_{k \mid k, m-1}^{Q}}{\sqrt{\mathbf{h}_{k m}^{j^{T}} \mathbf{P}_{k \mid k, m-1}^{Q} \mathbf{h}_{k m}^{j}+\sigma_{k m}^{j^{2}}}}
$$

Proof: The proof is similar to that of Proposition 1 and is presented in [16].

Proposition 3: H-estimator

Consider the linear model of (1)-(3). If robot $i$ assumes the pdf $p\left[\mathbf{x}_{k} \mid \mathbf{b}_{0: k, m-1}^{q \neq i}, \mathbf{z}_{0: k, m-1}^{i}\right]$ to be Gaussian with $\mathcal{N}\left(\hat{\mathbf{x}}_{k \mid k, m-1}^{H_{i}}, \mathbf{P}_{k \mid k, m-1}^{H_{i}}\right)$, then the MMSE estimator that processes the analog measurement, $z_{k m}^{i}$, is identical to the Kalman filter (see Algorithm 1). For the MMSE estimator that processes the quantized measurement, $b_{k m}^{j}, j \neq i$, the state and covariance update equations are given by

$$
\begin{gathered}
\hat{\mathbf{x}}_{k \mid k, m}^{H_{i}}=\hat{\mathbf{x}}_{k \mid k, m-1}^{H_{i}}+\frac{\left(\alpha_{H_{i}} / \sqrt{2 \pi}\right) \mathbf{P}_{k \mid k, m-1}^{H_{i}} \mathbf{h}_{k m}^{j}}{\sqrt{\mathbf{h}_{k m}^{j^{T}} \mathbf{P}_{k \mid k, m-1}^{H_{i}} \mathbf{h}_{k m}^{j}+\sigma_{k m}^{j^{2}}}} b_{k m}^{j} \\
\mathbf{P}_{k \mid k, m}^{H_{i}}=\mathbf{P}_{k \mid k, m-1}^{H_{i}}-\frac{\alpha_{H_{i}}^{2} \mathbf{P}_{k \mid k, m-1}^{H_{i}} \mathbf{h}_{k m}^{j} \mathbf{h}_{k m}^{j^{T}} \mathbf{P}_{k \mid k, m-1}^{H_{i}}}{2 \pi\left(\mathbf{h}_{k m}^{j^{T}} \mathbf{P}_{k \mid k, m-1}^{H_{i}} \mathbf{h}_{k m}^{j}+\sigma_{k m}^{j^{2}}\right)}
\end{gathered}
$$

where

$$
0 \leq \alpha_{H_{i}}=\frac{\exp \left[-\beta_{H_{i}}^{2} / 2\right]}{\mathrm{Q}\left[b_{k m}^{j} \beta_{H_{i}}\right]}
$$

and

$$
\beta_{H_{i}}=\frac{-\mathbf{d}^{T} \hat{\mathbf{x}}_{k \mid k, m-1}^{H_{j}}-\mathbf{c}^{T} \hat{\mathbf{x}}_{k \mid k, m-1}^{Q}-\mathbf{h}_{k m}^{j^{T}} \hat{\mathbf{x}}_{k \mid k, m-1}^{H_{i}}}{\sqrt{\mathbf{h}_{k m}^{j^{T}} \mathbf{P}_{k \mid k, m-1}^{H_{i}} \mathbf{h}_{k m}^{j}+\sigma_{k m}^{j^{2}}}}
$$

Proof: The proof is similar to that of Proposition 1 and is presented in [16].

1) Quantization bit encoding: Based on the general rule given in (9), we seek to determine the values of $\mathbf{c}$ and $\mathbf{d}$ that generate the most informative bit, $b_{k m}^{j}$. This is achieved by the following quantization rule (the details of the derivation are available in [16])

$$
b_{k m}^{j}:=\operatorname{sign}\left[\mathbf{h}_{k m}^{j^{T}}\left(\hat{\mathbf{x}}_{k \mid k, m}^{H_{j}}-\hat{\mathbf{x}}_{k \mid k, m-1}^{Q}\right)\right]
$$

where, $\hat{\mathbf{x}}_{k \mid k, m}^{H_{j}}=\hat{\mathbf{x}}_{k \mid k, m-1}^{H_{j}}+\mathbf{k}_{k m}^{H_{j}}\left(z_{k m}^{j}-\mathbf{h}_{k m}^{j^{T}} \hat{\mathbf{x}}_{k \mid k, m-1}^{H_{j}}\right)$, is the estimate generated by the H-estimator for robot $j$ after processing $z_{k m}^{j}$, and $\mathbf{k}_{k m}^{H_{j}}=$ $\mathbf{P}_{k \mid k, m-1}^{H_{j}} \mathbf{h}_{k m}^{j}\left(\mathbf{h}_{k m}^{j^{T}} \mathbf{P}_{k \mid k, m-1}^{H_{j}} \mathbf{h}_{k m}^{j}+\sigma_{k m}^{j^{2}}\right)^{-1}$. Therefore, (16) can be expressed as

$$
\begin{gathered}
b_{k m}^{j}:=\operatorname{sign}\left[z_{k m}^{j}+\frac{\left(1-\mathbf{h}_{k m}^{j^{T}} \mathbf{k}_{k m}^{H_{j}}\right) \mathbf{h}_{k m}^{j^{T}}}{\mathbf{h}_{k m}^{j^{T}} \mathbf{k}_{k m}^{H_{j}}} \hat{\mathbf{x}}_{k \mid k, m-1}^{H_{j}}\right. \\
\left.-\frac{\mathbf{h}_{k m}^{j^{T}}}{\mathbf{h}_{k m}^{j^{T}} \mathbf{k}_{k m}^{H_{j}}} \hat{\mathbf{x}}_{k \mid k, m-1}^{Q}\right]
\end{gathered}
$$

Comparing (17) with (9), we obtain the explicit values for $\mathbf{c}$ and $\mathbf{d}$ (see Table I). 
We now prove that the bit generated using this new quantization rule is indeed more informative than the SOI bit. Firstly, the SOI rule can be written as (see (2) and (5))

$$
\begin{aligned}
b_{k m}^{j} & :=\operatorname{sign}\left[\mathbf{h}_{k m}^{j^{T}} \mathbf{x}_{k}+v_{k m}^{j}-\mathbf{h}_{k m}^{j^{T}} \hat{\mathbf{x}}_{k \mid k, m-1}^{Q}\right] \\
& =\operatorname{sign}\left[\mathbf{h}_{k m}^{j^{T}} \tilde{\mathbf{x}}_{k}^{Q}+v_{k m}^{j}\right]
\end{aligned}
$$

where $\tilde{\mathbf{x}}_{k}^{Q}=\mathbf{x}_{k}-\hat{\mathbf{x}}_{k \mid k, m-1}^{Q}$. Similarly, the quantization rule in (16) can be written as

$$
b_{k m}^{j}:=\operatorname{sign}\left[\mathbf{h}_{k m}^{j^{T}} \tilde{\mathbf{x}}_{k}^{Q}+\mathbf{h}_{k m}^{j^{T}}\left(\hat{\mathbf{x}}_{k \mid k, m}^{H_{j}}-\mathbf{x}_{k}\right)\right]
$$

Ideally, in the noise-free case, the quantity that the SOI rule quantizes will be $\mathbf{h}_{k m}^{j^{T}} \tilde{\mathbf{x}}_{k}^{Q}$. Thus, if we show that the uncertainty of $\mathbf{h}_{k m}^{j^{T}}\left(\hat{\mathbf{x}}_{k \mid k, m}^{H_{j}}-\mathbf{x}_{k}\right)$, i.e., $\mathbf{h}_{k m}^{j^{T}} \mathbf{P}_{k \mid k, m}^{H_{j}} \mathbf{h}_{k m}^{j}$, is less than the uncertainty of $v_{k m}^{j}$, i.e., $\sigma_{k m}^{j^{2}}$, this implies that the bit generated using (16) is more informative than the SOI bit.

$$
\begin{aligned}
& \mathbf{h}_{k m}^{j^{T}} \mathbf{P}_{k \mid k, m}^{H_{j}} \mathbf{h}_{k m}^{j} \\
& =\mathbf{h}_{k m}^{j^{T}} \mathbf{P}_{k \mid k, m-1}^{H_{j}} \mathbf{h}_{k m}^{j}-\frac{\left(\mathbf{h}_{k m}^{j^{T}} \mathbf{P}_{k \mid k, m-1}^{H_{j}} \mathbf{h}_{k m}^{j}\right)^{2}}{\left(\mathbf{h}_{k m}^{j^{T}} \mathbf{P}_{k \mid k, m-1}^{H_{j}} \mathbf{h}_{k m}^{j}+\sigma_{k m}^{j^{2}}\right)} \\
& =\frac{\mathbf{h}_{k m}^{j^{T}} \mathbf{P}_{k \mid k, m-1}^{H_{j}} \mathbf{h}_{k m}^{j}}{\mathbf{h}_{k m}^{j^{T}} \mathbf{P}_{k \mid k, m-1}^{H_{j}} \mathbf{h}_{k m}^{j}+\sigma_{k m}^{j^{2}}} \sigma_{k m}^{j^{2}} \leq \sigma_{k m}^{j^{2}}
\end{aligned}
$$

Thus, by incorporating information from the H-estimator, the new quantization rule generates more informative bits than the SOI rule.

2) Quantization bit decoding: Let us now focus on how bit $b_{k m}^{j}$, generated by robot $j$ using the new quantization rule, is processed by the robots in the team. Ideally, in the absence of communication constraints, when another robot (say robot $i, i \neq j$ ) receives bit $b_{k m}^{j}$ from robot $j$, it would use the estimators presented in Propositions 2, 3 for processing this bit. However, communication constraints prevent robot $i$ from computing $\mathbf{c}$ and $\mathbf{d}$ in the following way: (i) The $\mathrm{H}$ and Q-estimators for robot $i$ depend on $\hat{\mathbf{x}}_{k \mid k, m-1}^{H_{j}}$, which is not available locally to robot $i$ (see (12), (15)). Therefore, robot $i$ has to set $\mathbf{d}=\mathbf{0}$. (ii) The parameter $\mathbf{c}$ in the quantization rule (see (17)) depends on the Kalman gain, $\mathbf{k}_{k m}^{H_{j}}$, of robot $j$. Again, this information is not available to robot $i$. Therefore, robot $i$ has to approximate $\mathbf{c}$ by a locally available quantity. We select $\mathbf{c}$ so that it minimizes the average uncertainty in the estimates generated by the Qestimator. This is achieved when $\beta_{Q}=0$ in (12) (for details see [16]), which corresponds to setting $\mathbf{c}=-\mathbf{h}_{k m}^{j}$. Note that $\mathbf{h}_{k m}^{j}$ is, in fact, locally available to robot $i$.

Substituting these choices for $\mathbf{c}$ and $\mathbf{d}$ (see Table I) in (10)(15), we see that the resulting estimators are identical to the $\mathrm{Q}$ and H-estimators corresponding to the SOI quantization rule (see Section III-A). This implies that by using the new quantization rule, robot $j$ can generate a more accurate bit

\begin{tabular}{|c|c|c|}
\hline & $\mathbf{c}^{T}$ & $\mathbf{d}^{T}$ \\
\hline Robot $j$ generating bit $b_{k m}^{j}$ & $-\frac{\mathbf{h}_{k m}^{j^{T}}}{\mathbf{h}_{k m}^{j^{T}} \mathbf{k}_{k m}^{H}}$ & $\frac{\left(1-\mathbf{h}_{k m}^{j^{T}} \mathbf{k}_{k m}^{H_{j}}\right) \mathbf{h}_{k m}^{j^{T}}}{\mathbf{h}_{k m}^{j^{T}} \mathbf{k}_{k m}^{H_{j}}}$ \\
\hline Robot $i$ processing bit $b_{k m}^{j}$ & $-\mathbf{h}_{k m}^{j^{T}}$ & 0 \\
\hline
\end{tabular}
than the SOI bit, but the communication constraints coupled with our design choice, force robot $i$ to decode and process the bit using the $\mathrm{H}$ and Q-estimators for the SOI quantization
TABLE I

CHOOSING $\mathbf{c}$ AND d FOR THE NEW QUANTIZATION RULE

rule. Algorithm 3 describes the proposed hybrid estimation framework using the new quantization rule. For clarity, we denote these Q-estimator and H-estimator based on the new quantization rule in (16) as M-SOI-KF and MH-SOI-KF respectively (see Fig. 1).

\section{Simulation Results}

The simulation set-up consists of a team of two robots navigating in $2 \mathrm{D}$ while performing $\mathrm{CL}$. The system model for each robot is given by the constant velocity motion model [15]. The continuous-time dynamics for each robot are given by

$$
\dot{\mathbf{x}}=\mathbf{f}(\mathbf{x})+\mathbf{G}_{c}\left[\begin{array}{l}
w_{V} \\
w_{\omega}
\end{array}\right]
$$

where $\mathbf{x}=[x, y, \phi, V, \omega]^{T}, \mathbf{f}(\mathbf{x})=$ $[V \cos \phi, V \sin \phi, \omega, 0,0]^{T}$ and $\mathbf{G}_{c}=\left[\mathbf{0}_{2 \times 3}, \mathbf{I}_{2 \times 2}\right]^{T}$. The standard deviation of the continuous-time noise in the linear, $V$, and rotational, $\omega$, velocity is chosen to be $\sigma_{V}=0.6325 \mathrm{~m} / \mathrm{s} \cdot \sqrt{\mathrm{Hz}}$ and $\sigma_{\omega}=0.4967 \mathrm{rad} / \mathrm{s} . \sqrt{\mathrm{Hz}}$ respectively.

The first-order discretized model with time-step $\delta t$ is given by, $\mathbf{x}_{k+1}=\mathbf{x}_{k}+\mathbf{f}\left(\mathbf{x}_{k}\right) \delta t+\mathbf{w}_{d}$, where $\mathbf{w}_{d}$ is the discrete-time noise, with $E\left[\mathbf{w}_{d} \mathbf{w}_{d}^{T}\right]=\mathbf{Q}_{d}$ and

$$
\mathbf{Q}_{d}=\int_{t_{k}}^{t_{k+1}} \boldsymbol{\Phi}\left(t_{k+1}, \tau\right) \mathbf{G}_{c} \mathbf{Q}_{c} \mathbf{G}_{c}^{T} \mathbf{\Phi}\left(t_{k+1}, \tau\right)^{T} d \tau
$$

with $\mathbf{Q}_{c}=\operatorname{diag}\left(\sigma_{V}^{2}, \sigma_{\omega}^{2}\right)$ and

$$
\boldsymbol{\Phi}_{k}=\left[\begin{array}{ccccc}
1 & 0 & -V_{k} \delta t \sin \phi_{k} & \delta t \cos \phi_{k} & 0 \\
0 & 1 & V_{k} \delta t \cos \phi_{k} & \delta t \sin \phi_{k} & 0 \\
0 & 0 & 1 & 0 & \delta t \\
0 & 0 & 0 & 1 & 0 \\
0 & 0 & 0 & 0 & 1
\end{array}\right]
$$

Each robot obtains measurements for its linear, $V_{m}$, and rotational, $\omega_{m}$, velocity, as well as its distance, $d_{m}$, and bearing, $\theta_{m}$, to the other robot. The noise in these measurements is modeled as zero-mean, white Gaussian with standard deviation $\sigma_{V_{m}}=0.07 \mathrm{~m} / \mathrm{s}, \sigma_{\omega_{m}}=0.28 \mathrm{rad} / \mathrm{s}$ for the linear and rotational velocity measurements, respectively, and $\sigma_{d_{m}}=0.05 \mathrm{~m}, \sigma_{\theta_{m}}=0.09 \mathrm{rad}$. for the corresponding distance and bearing measurements.

In this section, we compare the performance of the proposed: (i) H-SOI-EKF estimator based on the SOI quantization rule, and (ii) M-SOI-KF (quantized) and MH-SOI$\mathrm{KF}$ (hybrid) estimators obtained using the new quantization rule in (16), with that of the SOI-KF of [12] and the analog 


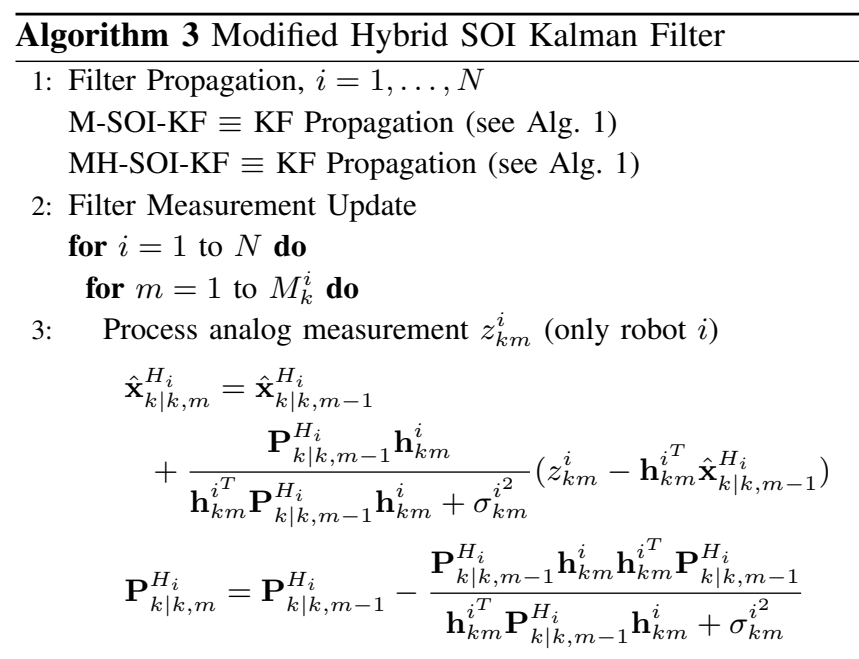

4: $\quad$ Generate quantization bit $b_{k m}^{i}$ (only robot $i$ )

$$
b_{k m}^{i}:=\operatorname{sign}\left[\mathbf{h}_{k m}^{i^{T}}\left(\hat{\mathbf{x}}_{k \mid k, m}^{H_{i}}-\hat{\mathbf{x}}_{k \mid k, m-1}^{Q}\right)\right]
$$

5: $\quad$ Process quantization bit $b_{k m}^{i}$

M-SOI-KF (all robots)

$$
\begin{aligned}
\hat{\mathbf{x}}_{k \mid k, m}^{Q}= & \hat{\mathbf{x}}_{k \mid k, m-1}^{Q}+\frac{\sqrt{2 / \pi} \mathbf{P}_{k \mid k, m-1}^{Q} \mathbf{h}_{k m}^{i}}{\sqrt{\mathbf{h}_{k m}^{i^{T}} \mathbf{P}_{k \mid k, m-1}^{Q} \mathbf{h}_{k m}^{i}+\sigma_{k m}^{i^{2}}}} b_{k m}^{i} \\
\mathbf{P}_{k \mid k, m}^{Q}= & \mathbf{P}_{k \mid k, m-1}^{Q} \\
& -\frac{(2 / \pi) \mathbf{P}_{k \mid k, m-1}^{Q} \mathbf{h}_{k m}^{i} \mathbf{h}_{k m}^{i^{T}} \mathbf{P}_{k \mid k, m-1}^{Q}}{\mathbf{h}_{k m}^{i^{T}} \mathbf{P}_{k \mid k, m-1}^{Q} \mathbf{h}_{k m}^{i}+\sigma_{k m}^{i^{2}}}
\end{aligned}
$$

MH-SOI-KF, $j=1, \ldots, N, j \neq i$

$$
\begin{aligned}
\hat{\mathbf{x}}_{k \mid k, m}^{H_{j}}= & \hat{\mathbf{x}}_{k \mid k, m-1}^{H_{j}}+\frac{\left(\alpha_{H_{j}} / \sqrt{2 \pi}\right) \mathbf{P}_{k \mid k, m-1}^{H_{j}} \mathbf{h}_{k m}^{i}}{\sqrt{\mathbf{h}_{k m}^{i^{T}} \mathbf{P}_{k \mid k, m-1}^{H_{j}} \mathbf{h}_{k m}^{i}+\sigma_{k m}^{i^{2}}}} b_{k m}^{i} \\
\mathbf{P}_{k \mid k, m}^{H_{j}}= & \mathbf{P}_{k \mid k, m-1}^{H_{j}} \\
& -\frac{\alpha_{H_{j}}^{2} \mathbf{P}_{k \mid k, m-1}^{H_{j}} \mathbf{h}_{k m}^{i} \mathbf{h}_{k m}^{i^{T}} \mathbf{P}_{k \mid k, m-1}^{H_{j}}}{2 \pi\left(\mathbf{h}_{k m}^{i^{T}} \mathbf{P}_{k \mid k, m-1}^{H_{j}} \mathbf{h}_{k m}^{i}+\sigma_{k m}^{i^{2}}\right)}
\end{aligned}
$$

where,

$$
0 \leq \alpha_{H_{j}}=\frac{\exp \left[-\beta_{H_{j}}^{2} / 2\right]}{\mathrm{Q}\left[b_{k m}^{i} \beta_{H_{j}}\right]}
$$

$$
\text { and } \beta_{H_{j}}=\frac{\mathbf{h}_{k m}^{i^{T}}\left(\hat{\mathbf{x}}_{k \mid k, m-1}^{Q}-\hat{\mathbf{x}}_{k \mid k, m-1}^{H_{j}}\right)}{\sqrt{\mathbf{h}_{k m}^{i^{T}} \mathbf{P}_{k \mid k, m-1}^{H_{j}} \mathbf{h}_{k m}^{i}+\sigma_{k m}^{i^{2}}}}
$$

end

$$
\begin{aligned}
& \hat{\mathbf{x}}_{k \mid k, 0}^{Q}:=\hat{\mathbf{x}}_{k \mid k, M_{k}^{i}}^{Q}, \mathbf{P}_{k \mid k, 0}^{Q}:=\mathbf{P}_{k \mid k, M_{k}^{i}}^{Q} \\
& \hat{\mathbf{x}}_{k \mid k, 0}^{H_{j}}:=\hat{\mathbf{x}}_{k \mid k, M_{k}^{i}}^{H_{j}}, \mathbf{P}_{k \mid k, 0}^{H_{j}}:=\mathbf{P}_{k \mid k, M_{k}^{i}}^{H_{j}}, j=1, \ldots, N \\
& \text { end } \\
& \hat{\mathbf{x}}_{k \mid k}^{Q}:=\hat{\mathbf{x}}_{k \mid k, M_{k}^{N}}^{Q}, \mathbf{P}_{k \mid k}^{Q}:=\mathbf{P}_{k \mid k, M_{k}^{N}}^{Q} \\
& \hat{\mathbf{x}}_{k \mid k}^{H_{j}}:=\hat{\mathbf{x}}_{k \mid k, M_{k}^{N}}^{H_{j}}, \mathbf{P}_{k \mid k}^{H_{j}}:=\mathbf{P}_{k \mid k, M_{k}^{N}}^{H_{j}}, \quad j=1, \ldots, N
\end{aligned}
$$

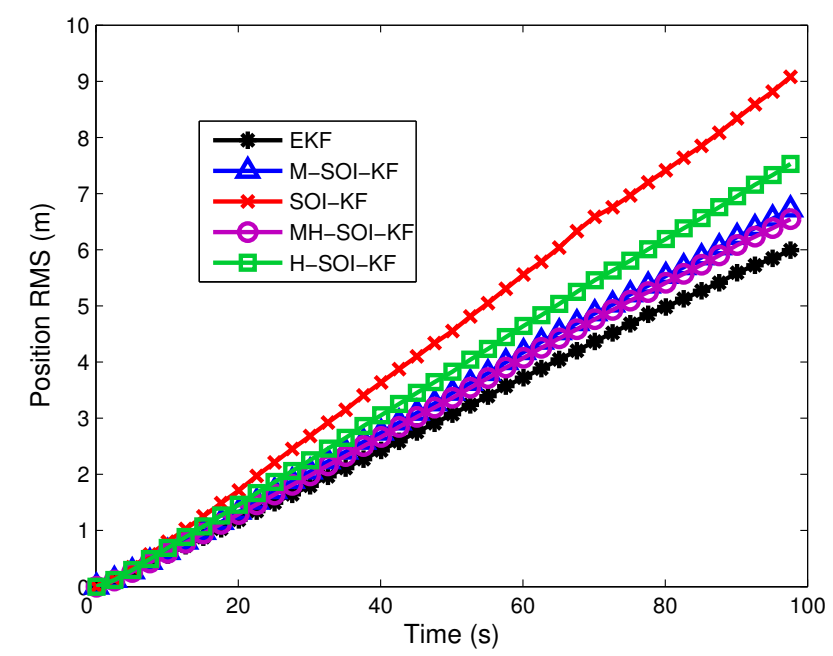

(a) Comparison of position RMS error.

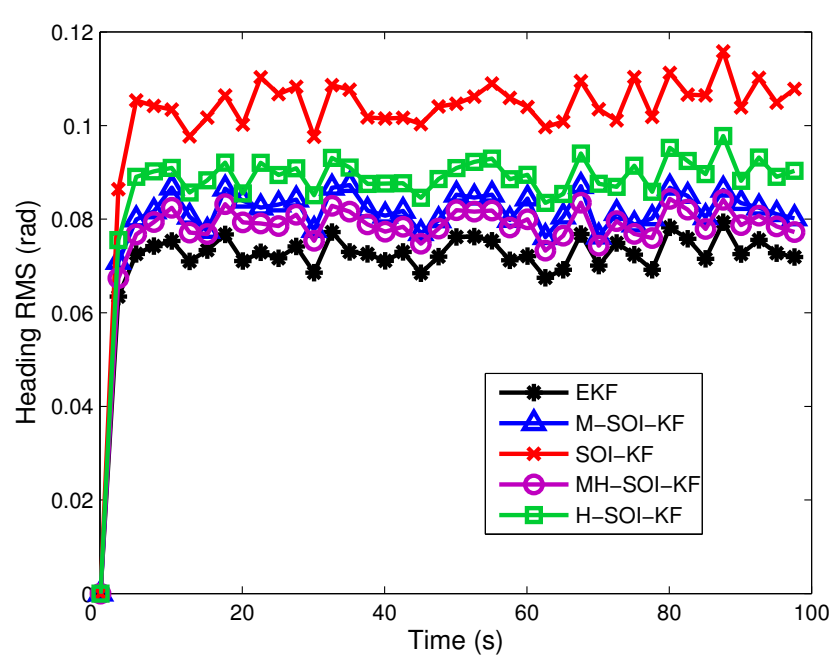

(b) Comparison of heading RMS error.

Fig. 2. Comparison of the RMS error for EKF, M-SOI-KF, SOI-KF, MHSOI-KF and H-SOI-KF estimators.

Extended Kalman filter (EKF) (that processes the actual realvalued measurements). Figures 2(a), 2(b) show the RMS error in the position and orientation estimates for these estimators, averaged over the 2 robots in the team and 100 Monte Carlo trials. Note that since the estimates generated by the $\mathrm{H}$-estimator for robot 1 are different from those generated by the $\mathrm{H}$-estimator for robot 2, the RMS errors for the $\mathrm{H}$ estimators, H-SOI-KF and MH-SOI-KF, are also averaged over estimators maintained by each robot.

First, let us compare the performance of the H and Qestimators designed using the SOI quantization rule. From Figs. 2(a), 2(b) we see that the estimates generated by the $\mathrm{H}-\mathrm{SOI}-\mathrm{KF}$ are more accurate than the SOI-KF estimates. This is because the H-SOI-KF, contrary to the SOI-KF, is able to incorporate information from the locally-available, analog measurements into the estimation process, resulting 
into improved estimation accuracy. Next, we focus on the estimators designed using the new quantization rule. Here, we observe that the modified Q-estimator, M-SOI-KF, that processes only quantized bits, outperforms not only the SOIKF but also the H-SOI-KF. Furthermore, as expected, the modified $\mathrm{H}$-estimator, $\mathrm{MH}-\mathrm{SOI}-\mathrm{KF}$, that also processes information from locally-available analog measurements, outperforms the M-SOI-KF. Thus, we see that by incorporating information from the estimates generated by the $\mathrm{H}$-estimator into the quantization rule, we improve the performance of both modified estimators. Lastly, even though each robot can communicate only a single bit per real-valued measurement, we note that the performance obtained by using the new quantization rule is comparable to that of the EKF (where each robot communicates the actual real-valued measurements).

\section{CONClusions And Future Work}

In this paper, we presented a hybrid estimation framework for multi-centralized CL under the constraint that each robot communicates only a single bit per scalar measurement. The proposed approach, where each robot maintains a quantized and a hybrid estimator, improves the accuracy of the robots' pose estimates by enabling each robot to incorporate information from its locally-available, analog measurements into the estimation process. Moreover, we showed that by modifying the quantization rule to include information from the hybrid estimator, the performance of both the quantized and hybrid estimators can be further improved.

As a part of our future work, we will work on analytically characterizing the performance gain of the hybrid estimation framework. Also, we will extend our proposed approach to the case when more than one bit can be transmitted per scalar measurement. Finally, since the process and measurement models in robotics are, in general, non-linear, we will develop an extension of this approach to the MAP estimation framework so as to improve the accuracy of the robots' pose estimates.

\section{REFERENCES}

[1] T. L. Huntsberger, A. Trebi-Ollennu, H. Aghazarian, P. S. Schenker, P. Pirjanian, and H. D. Nayar, "Distributed control of multi-robot systems engaged in tightly coupled tasks," Autonomous Robots, vol. 17, no. 1, pp. 79-92, 2004.

[2] Y. Feng, Z. Zhu, and J. Xiao, "Heterogeneous multi-robot localization in unknown 3D space," in Proc. of the IEEE/RSJ International Conference on Intelligent Robots and Systems, Beijing, China, Oct. 9-15, 2006, pp. 4533-4538.

[3] A. D. Tews, G. S. Sukhatme, and M. J. Mataric, "A multi-robot approach to stealthy navigation in the presence of an observer," in Proc. of the IEEE International Conference on Robotics and Automation, New Orleans, LA, Apr. 26-May 1, 2004, pp. 2379-2385.

[4] I. M. Rekleitis, G. Dudek, and E. E. Milios, "Multi-robot collaboration for robust exploration," in Proc. of the IEEE International Conference on Robotics and Automation, San Francisco, CA, Apr. 24-28, 2000, pp. 3164-3169.

[5] S. Roumeliotis and G. Bekey, "Distributed multirobot localization," IEEE Transactions on Robotics and Automation, vol. 18, no. 5, pp. 781-795, Oct. 2002.

[6] K. Y. K. Leung, T. D. Barfoot, and H. Liu, "Decentralized localization of sparsely-communicating robot networks: A centralized-equivalent approach," IEEE Transactions on Robotics, vol. 26, no. 1, pp. 62-77, Feb. 2010 .
[7] A. I. Mourikis and S. I. Roumeliotis, "Optimal sensor scheduling for resource-constrained localization of mobile robot formations," IEEE Transactions on Robotics, vol. 22, no. 5, pp. 917-931, Oct. 2006.

[8] E. D. Nerurkar and S. I. Roumeliotis, "Asynchronous multi-centralized cooperative localization," in Proc. of the IEEE/RSJ International Conference on Intelligent Robots and Systems, Taipei, Taiwan, Oct. 18-22, 2010, pp. 4352-4359.

[9] W.-M. Lam and A. R. Reibman, "Design of quantizers for decentralized estimation systems," IEEE Transactions on Communications, vol. 41, no. 11, pp. 1602-1605, Nov. 1993.

[10] H. C. Papadopoulos, G. W. Wornell, and A. V. Oppenheim, "Sequential signal encoding from noisy measurements using quantizers with dynamic bias control," IEEE Transactions on Information Theory, vol. 47, no. 3, pp. 978-1002, Mar. 2001.

[11] F. A. Shah, A. Ribeiro, and G. B. Giannakis, "Bandwidth-constrained map estimation for wireless sensor networks," in Proc. of Thirty-Ninth Asilomar Conference on Signals, Systems and Computers, Pacific Grove, CA, Oct. 30-Nov. 2, 2005, pp. 215-219.

[12] A. Ribeiro, G. B. Giannakis, and S. I. Roumeliotis, "SOI-KF: Distributed kalman filtering with low-cost communications using the sign of innovations," IEEE Transactions on Signal Processing, vol. 54, no. 12, pp. 4782-4795, Dec. 2006.

[13] E. J. Msechu, S. I. Roumeliotis, A. Ribeiro, and G. B. Giannakis, "Decentralized quantized kalman filtering with scalable communication cost," IEEE Transactions on Signal Processing, vol. 56, no. 8, pp. 3727-3741, Aug. 2008.

[14] N. Trawny, S. I. Roumeliotis, and G. B. Giannakis, "Cooperative multirobot localization under communication constraints," in Proc. of the IEEE International Conference on Robotics and Automation, Kobe, Japan, May 12-17, 2009, pp. 4394-4400.

[15] Y. Bar-Shalom and X.-R. Li, Estimation and Tracking: principles, techniques and software. Norwood, MA: Artech House, Inc., 1993.

[16] E. D. Nerurkar, K. X. Zhou, and S. I. Roumeliotis, "Hybrid estimation framework of multi-robot cooperative localization using quantized measurements," Dept. of Comp. Sci. \& Eng., University of Minnesota, Tech. Rep., 2011. [Online]. Available: http://wwwusers.cs.umn.edu/ n nerurkar/Nerurkar_hybridestimationCL.pdf

[17] P. S. Maybeck, Stochastic models, estimation, and control, ser. Mathematics in Science and Engineering, 1979, vol. 141.

\section{APPENDIX}

Under the Gaussian assumption for the pdf $p\left[\mathbf{x}_{k} \mid \mathbf{b}_{0: k, m-1}^{q \neq i}, \mathbf{z}_{0: k, m-1}^{i}\right]$, the state and covariance propagation derivation is straightforward and identical to that of the standard Kalman filter [17].

The MMSE estimate for robot $i$ 's H-estimator, obtained after processing bit $b_{k m}^{j}$, is defined as

$$
\begin{aligned}
\hat{\mathbf{x}}_{k \mid k, m}^{H_{i}} & =E\left[\mathbf{x}_{k} \mid \mathbf{b}_{0: k, m}^{q \neq i}, \mathbf{z}_{0: k, m-1}^{i}\right] \\
& =\int_{\mathbb{R}^{p}} \mathbf{x}_{k} p\left[\mathbf{x}_{k} \mid \mathbf{b}_{0: k, m}^{q \neq i}, \mathbf{z}_{0: k, m-1}^{i}\right] d \mathbf{x}_{k}
\end{aligned}
$$

Using Bayes' rule, we can express the posterior pdf as

$$
\begin{aligned}
& p\left[\mathbf{x}_{k} \mid \mathbf{b}_{0: k, m-1}^{q \neq i}, b_{k m}^{j}, \mathbf{z}_{0: k, m-1}^{i}\right] \\
& =\frac{p\left[b_{k m}^{j} \mid \mathbf{x}_{k}, \mathbf{b}_{0: k, m-1}^{q \neq i}, \mathbf{z}_{0: k, m-1}^{i}\right] p\left[\mathbf{x}_{k} \mid \mathbf{b}_{0: k, m-1}^{q \neq i}, \mathbf{z}_{0: k, m-1}^{i}\right]}{p\left[b_{k m}^{j} \mid \mathbf{b}_{0: k, m-1}^{q \neq i}, \mathbf{z}_{0: k, m-1}^{i}\right]}
\end{aligned}
$$

where $\mathbf{b}_{0: k, m}^{q \neq i}=\left\{\mathbf{b}_{0: k, m-1}^{q \neq i}, b_{k m}^{j}\right\}, \mathbf{b}_{0: k, m-1}^{q \neq i}$ are the quantized measurements that have already been processed by robot $i$, and $b_{k m}^{j}$ is the new quantized measurement received by robot $i$. In (24), the term $p\left[b_{k m}^{j} \mid \mathbf{x}_{k}, \mathbf{b}_{0: k, m-1}^{q \neq i}, \mathbf{z}_{0: k, m-1}^{i}\right]$ can be expressed in terms of the Gaussian tail probability as 
follows

$$
\begin{aligned}
& p\left[b_{k m}^{j} \mid \mathbf{x}_{k}, \mathbf{b}_{0: k, m-1}^{q \neq i}, \mathbf{z}_{0: k, m-1}^{i}\right] \\
& :=\operatorname{Pr}\left\{b_{k m}^{j}= \pm 1 \mid \mathbf{x}_{k}, \mathbf{b}_{0: k, m-1}^{q \neq i}, \mathbf{z}_{0: k, m-1}^{i}\right\} \\
& =\operatorname{Pr}\left\{z_{k m}^{j}-\mathbf{h}_{k m}^{j^{T}} \hat{\mathbf{x}}_{k \mid k, m-1}^{Q} \gtrless 0 \mid \mathbf{x}_{k}\right\} \\
& =\operatorname{Pr}\left\{\mathbf{h}_{k m}^{j^{T}} \mathbf{x}_{k}+v_{k m}^{j}-\mathbf{h}_{k m}^{j^{T}} \hat{\mathbf{x}}_{k \mid k, m-1}^{Q} \gtrless 0 \mid \mathbf{x}_{k}\right\} \\
& =\operatorname{Pr}\left\{v_{k m}^{j} \gtrless-\mathbf{h}_{k m}^{j^{T}} \mathbf{x}_{k}+\mathbf{h}_{k m}^{j^{T}} \hat{\mathbf{x}}_{k \mid k, m-1}^{Q} \mid \mathbf{x}_{k}\right\} \\
& =\mathrm{Q}\left[b_{k m}^{j}\left(\frac{-\mathbf{h}_{k m}^{j^{T}} \mathbf{x}_{k}+\mathbf{h}_{k m}^{j^{T}} \hat{\mathbf{x}}_{k \mid k, m-1}^{Q}}{\sigma_{k m}^{j}}\right)\right]
\end{aligned}
$$

where the second equality results from the definition of the SOI quantization rule and the fact that since $\mathbf{x}_{k}$ is given, the conditioning on $\mathbf{b}_{0: k, m-1}^{q \neq i}, \mathbf{z}_{0: k, m-1}^{i}$ can be ignored. The next equality is obtained from the definition of $z_{k m}^{j}$ in (2). Since $v_{k m}^{j} \sim \mathcal{N}\left(0, \sigma_{k m}^{j^{2}}\right)$, we obtain the last equality from the definition of the Gaussian tail probability distribution ${ }^{4}$.

Similarly, the term $p\left[b_{k m}^{j} \mid \mathbf{b}_{0: k, m-1}^{q \neq i}, \mathbf{z}_{0: k, m-1}^{i}\right]$ can also be expressed in terms of the Gaussian tail probability as

$$
\begin{aligned}
& \operatorname{Pr}\left\{b_{k m}^{j}= \pm 1 \mid \mathbf{b}_{0: k, m-1}^{q \neq i}, \mathbf{z}_{0: k, m-1}^{i}\right\} \\
& =\operatorname{Pr}\left\{z_{k m}^{j} \gtrless \mathbf{h}_{k m}^{j^{T}} \hat{\mathbf{x}}_{k \mid k, m-1}^{Q} \mid \mathbf{b}_{0: k, m-1}^{q \neq i}, \mathbf{z}_{0: k, m-1}^{i}\right\} \\
& =\mathrm{Q}\left[b_{k m}^{j}\left(\frac{\mathbf{h}_{k m}^{j^{T}} \hat{\mathbf{x}}_{k \mid k, m-1}^{Q}-\mathbf{h}_{k m}^{j^{T}} \hat{\mathbf{x}}_{k \mid k, m-1}^{H_{i}}}{\sqrt{\mathbf{h}_{k m}^{j^{T}} \mathbf{P}_{k \mid k, m-1}^{H_{i}} \mathbf{h}_{k m}^{j}+\sigma_{k m}^{j^{2}}}}\right)\right] \triangleq \delta_{Q_{j}}
\end{aligned}
$$

where, $\quad p\left[z_{k m}^{j} \mid \mathbf{b}_{0: k, m-1}^{q \neq i}, \mathbf{z}_{0: k, m-1}^{i}\right]$ $\mathcal{N}\left(\mathbf{h}_{k m}^{j^{T}} \hat{\mathbf{x}}_{k \mid k, m-1}^{H_{i}}, \mathbf{h}_{k m}^{j^{T}} \mathbf{P}_{k \mid k, m-1}^{H_{i}} \mathbf{h}_{k m}^{j}+\sigma_{k m}^{j^{2}}\right)$.

Note that due to quantization, the pdf $p\left[\mathbf{x}_{k} \mid \mathbf{b}_{0: k, m-1}^{q \neq i}, \mathbf{z}_{0: k, m-1}^{i}\right] \quad$ is no longer Gaussian and calculating this pdf is, in general, intractable. Therefore, by approximating it as a Gaussian with $\mathcal{N}\left(\hat{\mathbf{x}}_{k \mid k, m-1}^{H_{i}}, \mathbf{P}_{k \mid k, m-1}^{H_{i}}\right)$ and from (23)- (26), we obtain

$$
\begin{array}{rl}
\hat{\mathbf{x}}_{k \mid k, m}^{H_{i}}=\frac{1}{\delta_{Q_{j}}} \int_{\mathbb{R}^{p}} \mathbf{x}_{k} & \mathbf{Q}\left[b_{k m}^{j}\left(\frac{-\mathbf{h}_{k m}^{j^{T}} \mathbf{x}_{k}+\mathbf{h}_{k m}^{j^{T}} \hat{\mathbf{x}}_{k \mid k, m-1}^{Q}}{\sigma_{k m}^{j}}\right)\right] \\
& \times \mathcal{N}\left(\hat{\mathbf{x}}_{k \mid k, m-1}^{H_{i}}, \mathbf{P}_{k \mid k, m-1}^{H_{i}}\right) d \mathbf{x}_{k}
\end{array}
$$

We obtain (7) after evaluating (27), the details of which

${ }^{4} Q[x] \triangleq \frac{1}{\sqrt{2 \pi}} \int_{x}^{\infty} \exp \left(-u^{2} / 2\right) d u$. If $Y \sim \mathcal{N}\left(\mu, \sigma^{2}\right)$, then $\operatorname{Pr}\{Y>y\}=\widehat{Q}[(y-\mu) / \sigma]$. are available in [16]. To obtain the covariance update equation in (8), we first define $\hat{\mathbf{x}}_{k \mid k, m}^{H_{i}}=\hat{\mathbf{x}}_{k \mid k, m-1}^{H_{i}}+$ $\mathbf{k}_{k m}^{H_{i}} b_{k m}^{j}$, where $\mathbf{k}_{k m}^{H_{i}}$ can be obtained from (7). Therefore, $\mathbf{P}_{k \mid k, m}^{H_{i}}:=E\left[\left(\mathbf{x}_{k}-\hat{\mathbf{x}}_{k \mid k, m}^{H_{i}}\right)\left(\mathbf{x}_{k}-\hat{\mathbf{x}}_{k \mid k, m}^{H_{i}}\right)^{T}\right]$, can be expressed as

$$
\begin{aligned}
\mathbf{P}_{k \mid k, m}^{H_{i}}=E & {\left[\left(\mathbf{x}_{k}-\hat{\mathbf{x}}_{k \mid k, m-1}^{H_{i}}-\mathbf{k}_{k m}^{H_{i}} b_{k m}^{j}\right)\right.} \\
& \left.\times\left(\mathbf{x}_{k}-\hat{\mathbf{x}}_{k \mid k, m-1}^{H_{i}}-\mathbf{k}_{k m}^{H_{i}} b_{k m}^{j}\right)^{T}\right] \\
= & \mathbf{P}_{k \mid k, m-1}^{H_{i}}+\mathbf{k}_{k m}^{H_{i}} \mathbf{k}_{k m}^{H_{i}^{T}} E\left[\left(b_{k m}^{j}\right)^{2}\right] \\
& -2 \mathbf{k}_{k m}^{H_{i}} E\left[b_{k m}^{j}\left(\mathbf{x}_{k}-\hat{\mathbf{x}}_{k \mid k, m-1}^{H_{i}}\right)^{T}\right]
\end{aligned}
$$

where $\mathbf{P}_{k \mid k, m-1}^{H_{i}}:=E\left[\left(\mathbf{x}_{k}-\hat{\mathbf{x}}_{k \mid k, m-1}^{H_{i}}\right)\left(\mathbf{x}_{k}-\hat{\mathbf{x}}_{k \mid k, m-1}^{H_{i}}\right)^{T}\right]$. The last term in (28) can be further simplified to obtain

$$
\begin{aligned}
E & {\left[b_{k m}^{j}\left(\mathbf{x}_{k}-\hat{\mathbf{x}}_{k \mid k, m-1}^{H_{i}}\right)^{T}\right] } \\
= & E\left[\left(\mathbf{x}_{k}-\hat{\mathbf{x}}_{k \mid k, m-1}^{H_{i}}\right)^{T} \mid b_{k m}^{j}=1\right] \operatorname{Pr}\left\{b_{k m}^{j}=1\right\} \\
& \quad-E\left[\left(\mathbf{x}_{k}-\hat{\mathbf{x}}_{k \mid k, m-1}^{H_{i}}\right)^{T} \mid b_{k m}^{j}=-1\right] \operatorname{Pr}\left\{b_{k m}^{j}=-1\right\} \\
= & \mathbf{k}_{k m}^{H_{i}^{T}} \operatorname{Pr}\left\{b_{k m}^{j}=1\right\}-\left(-\mathbf{k}_{k m}^{H_{i}^{T}}\right) \operatorname{Pr}\left\{b_{k m}^{j}=-1\right\} \\
= & \mathbf{k}_{k m}^{H_{i}^{T}}
\end{aligned}
$$

Thus, by substituting (29) in (28), along with the fact that $E\left[\left(b_{k m}^{j}\right)^{2}\right]=1$, we obtain (8).

We now consider the MMSE estimator for processing the analog measurement $z_{k m}^{i}$. The MMSE estimate for robot $i$ 's $\mathrm{H}$-estimator, obtained after processing $z_{k m}^{i}$, is

$$
\begin{aligned}
\hat{\mathbf{x}}_{k \mid k, m}^{H_{i}} & =E\left[\mathbf{x}_{k} \mid \mathbf{b}_{0: k, m-1}^{q \neq i}, \mathbf{z}_{0: k, m}^{i}\right] \\
& =\int_{\mathbb{R}^{p}} \mathbf{x}_{k} p\left[\mathbf{x}_{k} \mid \mathbf{b}_{0: k, m-1}^{q \neq i}, \mathbf{z}_{0: k, m}^{i}\right] d \mathbf{x}_{k}
\end{aligned}
$$

Using Bayes' rule, we can express the posterior pdf as

$$
\begin{aligned}
& p\left[\mathbf{x}_{k} \mid \mathbf{b}_{0: k, m-1}^{q \neq i}, \mathbf{z}_{0: k, m-1}^{i}, z_{k m}^{i}\right] \\
& =\frac{p\left[z_{k m}^{i} \mid \mathbf{x}_{k}, \mathbf{b}_{0: k, m-1}^{q \neq i}, \mathbf{z}_{0: k, m-1}^{i}\right] p\left[\mathbf{x}_{k} \mid \mathbf{b}_{0: k, m-1}^{q \neq i}, \mathbf{z}_{0: k, m-1}^{i}\right]}{p\left[z_{k m}^{i} \mid \mathbf{b}_{0: k, m-1}^{q \neq i}, \mathbf{z}_{0: k, m-1}^{i}\right]}
\end{aligned}
$$

Under the assumption that the pdf $p\left[\mathbf{x}_{k} \mid \mathbf{b}_{0: k, m-1}^{q \neq i}, \mathbf{z}_{0: k, m-1}^{i}\right] \quad$ is Gaussian, the pdfs $\quad p\left[z_{k m}^{i} \mid \mathbf{x}_{k}, \mathbf{b}_{0: k, m-1}^{q \neq i}, \mathbf{z}_{0: k, m-1}^{i}\right] \quad$ and $p\left[z_{k m}^{i} \mid \mathbf{b}_{0: k, m-1}^{q \neq i}, \mathbf{z}_{0: k, m-1}^{i}\right]$ also become Gaussians, similar to the Kalman filter. Thus, the derivation for the state and covariance update, from this point onwards, is identical to the Kalman filter and can be found in [17]. 